\title{
Impact of enhanced haematology palliative care services in patients with myelodysplastic syndrome and acute myeloid leukaemia: study protocol for a randomized controlled trial
}

\author{
Kwok Ying Chan ${ }^{1}$, Harinder Gill ${ }^{2,3}$, Cho Wing Li ${ }^{1}$, Thomas Sau Yan Chan ${ }^{2}$, Ho Yan Au ${ }^{1}$, Chi Yan Wong ${ }^{1}$, \\ Kwok Wai Tsang ${ }^{1}$, Raymond See Kit Lo ${ }^{4}$, Benjamin Hon Wai Cheng ${ }^{5} \wedge$ \\ ${ }^{1}$ Palliative Medical Unit, Grantham Hospital, Hong Kong, China; ${ }^{2}$ Division of Haematology, Department of Medicine, Queen Mary Hospital, Hong \\ Kong, China; ${ }^{3}$ Department of Medicine, University of Hong Kong, Hong Kong, China; ${ }^{4}$ Department of Palliative Medicine, Shatin Hospital, Hong \\ Kong, China; ${ }^{5}$ Medical Palliative Medicine Team, Department of Medicine and Geriatric, Tuen Mun Hospital, Hong Kong, China \\ Correspondence to: Dr. Kwok Ying Chan, MRCP, FHKCP. Palliative Medical Unit, Grantham Hospital, 125 Wong Chuk Hang, Hong Kong, China. \\ Email: cky842@yahoo.com.hk.
}

Background Patients with acute myeloid leukaemia (AML) and myelodysplastic syndrome (MDS) suffer
from a significant symptom burden and psychological, spiritual, social needs comparable to patients with
solid metastatic malignancy. Referral to palliative care services for these haematological patients remains
limited or often confined to the last days of life. We pioneered a palliative care (PC) program integrated
with standard haematological care. The purpose of this trial will study the interventions by the PC team and
preliminary results in the clinical outcomes.

Methods: This project is a non-blinded, randomized, controlled trial. In this study, we examine the clinical outcomes of the integrated PC program for MDS/AML patients when the 2 nd lines disease treatment failed and in the presence of prognostic indicators. In group 1, patients will receive standard haematological care associated with PC (i.e., intervention group). In contrast, in group 2, patients will receive standard haematological care only (i.e., control group) with PC service only on a request basis. Patients who join the program would have to complete a standardized questionnaire to assess their quality of life and their psychological and physical symptoms.

Results: This is to exam the impact of the early integrated palliative care with enhanced psychosocial interventions to both advanced MDS/AML patients and their primary family members in Hong Kong.

Discussion: This protocol will not display any result. If future results demonstrate that the enhanced PC interventions are effective, they will provide a quality treatment plan for patients with MDS/AML.

Trial Registration: The Hong Kong University/Hospital Authority Hong Kong West Institutional Review Board (HKU/HA HKW IRB). The registration number is UW 19-824.

Keywords! Early PC; haematology palliative care; randomized controlled trials (RCT)

Submitted Feb 02, 2021. Accepted for publication Jul 13, 2021.

doi: 10.21037/apm-20-1633

View this article at: https://dx.doi.org/10.21037/apm-20-1633

\footnotetext{
^ ORCID: Harinder Gill, 0000-0002-9551-4893; Benjamin Hon Wai Cheng, 0000-0002-5302-1804.
} 


\section{Introduction}

Acute myeloid leukaemia (AML) and myelodysplastic syndrome (MDS) are heterogeneous haematological cancer associated with poor long-term outcomes. The 5 -year overall survival is approximately $40 \%$ in AML patients $(1,2)$. Refractory/relapsed condition is seen in up to $40 \%$ of patients with AML and is often associated with grave prognosis, especially for those not fit for disease treatment (1). High-risk MDS is often incurable except for those patients eligible for haematopoietic stem transplantation (HSCT). MDS patients who failed first-line disease treatment have median survivals of less than six months (3). The majority of patients with AML and MDS are often diagnosed after the age of 65 , making them physically ineligible for intensive treatment (2). In addition to inferior survivals, most patients with relapsed or refractory AML and MDS have profound cytopenia resulting in an increased risk of infections and require frequent blood product support. Because of significant symptom burden, treatment side-effects, and repeated admission, their quality of life (QOL) is often badly affected. In addition, there was an associated heavy caregiver burden. Patients with AML and MDS often have a heavy symptom burden similar to that in patients with solid organ cancers (4-7).

The benefits of integrating early palliative care (PC) concurrently with standard care in solid cancer patients had shown in several major randomized controlled trials (RCT) (8-12). The majority of the studies revealed that the patients' mood and their QOL were improved, reducing healthcare cost at the end of life (EOL). Nowadays, it is recommended that those patients with advanced solid cancer receive PC early along the disease course during the disease (8). Their family members might also require support from the PC team (8).

From current evidence, the historical prioritization of cancer care at the center of palliative medicine did not guarantee that those diagnosed with a hematological malignancy were assured of timely/early referral. A key concern has been that patients with a hematological malignancy were most likely to end life amid escalating technology searching for a cure without access to PC (13). Our previous studies demonstrated that most patients with AML receive PC late and spend most of their endof-life periods in acute hospitals (14). In addition, there is a lack of studies examining the impact of early dedicated multidisciplinary PC services on HM patients' QOL, mood, and caregiver burden.
We present the following article in accordance with the SPIRIT reporting checklist (available at https://dx.doi. org/10.21037/apm-20-1633).

\section{Methods}

The primary objective is to evaluate the impact on patient QOL, mood, and caregiver burden by the interventions of Enhanced haematology palliative care (EHPC) when compared with standard haematology care alone on patients with MDS/AML.

\section{Design}

This is a prospective open-label RCT.

\section{The setting of the study}

The proposed study period will last for 24 months. Eligible patients with MDS and AML will be recruited to this nonblinded, RCT of EHPC integrated into hematology standard care versus standard care alone. The study would be performed at Queen Mary Hospital (QMH) of the Hong Kong West Cluster. Consenting patients will be randomly assigned to one of the two groups in a 1:1 ratio without stratification (Figure 1). Randomization will be performed by independent research staff. The randomization uses the minimization method to make an equal number between groups in the following items, including sex, age, and date of diagnosis (15).

\section{Participants}

Patients would be referred and included in the study if they fulfilled the following criteria

* Patients with AML or MDS according to the WHO 2016 criteria (16);

* Patients who fail a second or further line of treatment;

* Patients with AML or MDS with expected survivals of 6 months or less;

* Patients able to give an informed consent;

* Age $\geq 18$ years old;

* Cantonese speaking Chinese patient.

\section{Exclusion criteria}

Patients who could not give consent because of the language barrier or mental incapacity, those with pre-existing 


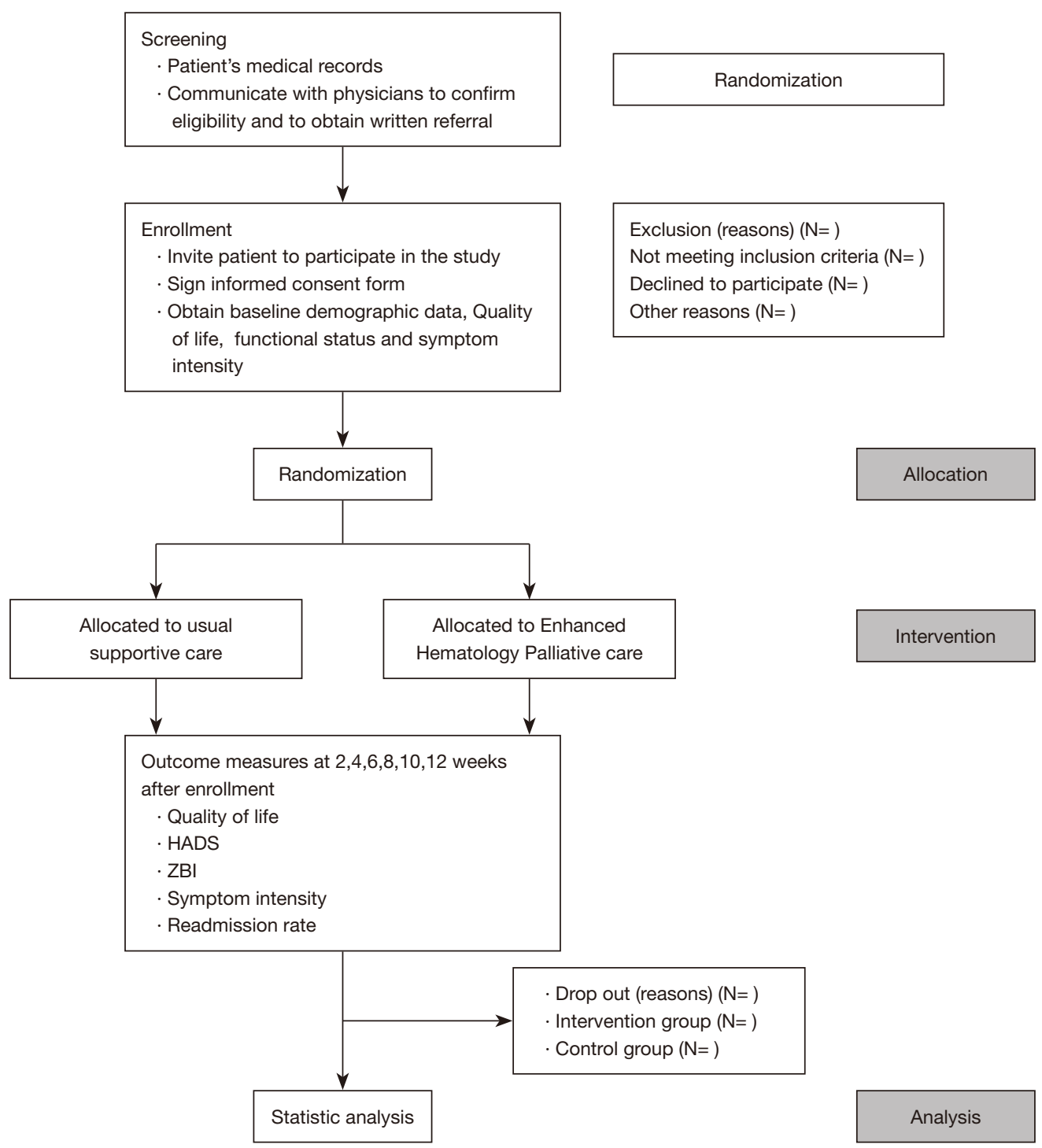

Figure 1 Flowchart showing the randomization of patients/caregivers to receive enhanced hematology palliative care or standard care (control group).

psychiatric illness or significant risk, will be excluded. Informed consent was taken. Then the research assistant (RA) proceeded to conduct a face-to-face interview with the patients using questionnaires.

\section{Randomization}

The RA who was blinded to the study would perform the baseline assessment. Before the assessment, the randomization was concealed within sealed envelopes. And following randomization, both the participants and main caregivers would know the group allocation.

\section{Intervention group}

Within 2 days of enrollment, patients assigned to EHPC ("fast-track" group) will be assessed at the outpatient clinic by the PC team. Thereafter, the patients will be assessed every two weeks until 12 weeks. The haematology PC team members will assess and follow-up patients at outpatient clinics, during admissions, and during home care visits. The onsite PC physician and nurse will provide symptom and psychosocial care, including any caring issue by 
Table 1 The details of the enhanced hematology palliative care program

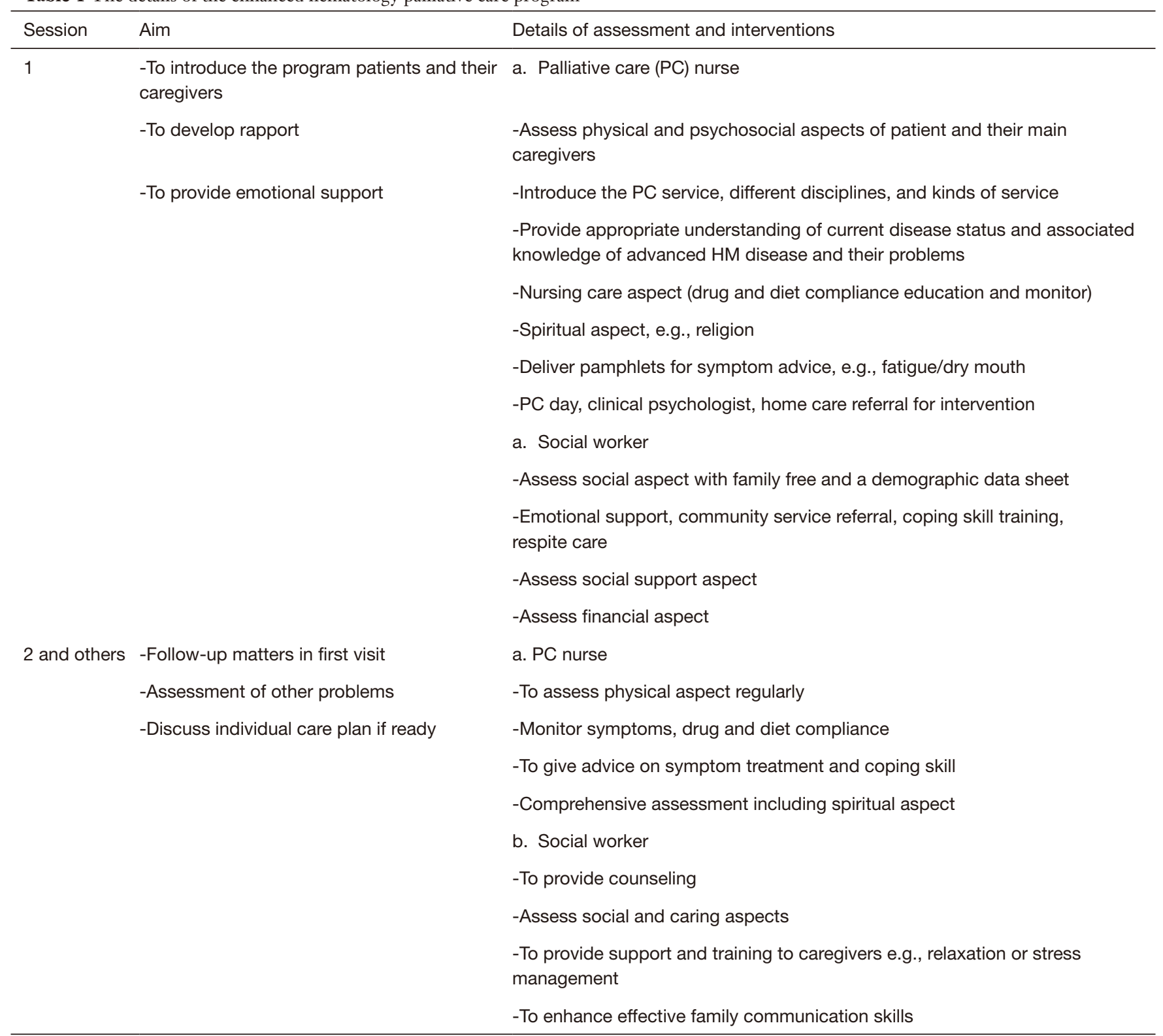

offering home care visits, telephone interviews, referring to appropriate community services, and discussing patients' needs at team meeting. The PC model for advanced hematology patients and its feasibility has been examined in our previous local study (17). Outpatients might be admitted to our PC in-patient care if clinically indicated. Referrals to physiotherapists, occupational therapists, speech therapists, dietitians, clinical psychologists, and bereavement councilors will be decided by the physician in charge based on the PC needs of patients and their families. The hospice daycare center will provide rehabilitation services, manage and monitor symptoms, and provide psychosocial support following discharge of patients from the hospital if required. Those still on palliative chemotherapy/disease treatment will remain in the intervention group. The components and objectives of care in the "fast-track" group are listed in Table 1. The number of home care and phone consultations will be evaluated at the end of the study.

Information will be gathered through individual interviews. The prompts are defined in advance, and 
researchers will explore the acceptability of the intervention giving the opportunity to the receivers (patients and family members) and the haematologists, involved in early integrated palliative care, to tell their experience.

\section{Control group}

Those allocated to the control group (i.e., standard care) will be taken care of by hematology team. Patients with special rehabilitation and social needs will be referred to physiotherapists, occupational therapists, and medical social workers. They will be admitted to the Haematology/ Oncology ward at QMH for in-patient care for symptom management as indicated. Patients in the control group will switch to receiving PC service after 12 weeks of care and be assessed every two weeks the same as the "fast-track" group (Figure 1). We chose 12 weeks because this will give enough time for both the conventional care and the EHPC to exert their effects on patients' quality of life, and a large proportion of patients from the conventional care group will be able to join the haematology PC services before they die (17). The reason to continue the study after 12 weeks of conventional care is to evaluate for any changes in clinical outcomes by receiving palliative care service as compared with conventional care. Longitudinal changes will be analyzed in terms of QOL, mood, and caregiver burden.

\section{Follow-up period}

RA will interview all subjects and administer the questionnaires to them in both intervention and control groups. Each subject was assessed every two weeks with the questionnaires until 12 weeks. Caregivers will be invited to participate in the interviews to obtain collateral information. The patients will complete self-rated questionnaires. The in-patient and outpatient data will be analyzed separately.

We chose to conduct a "wait-list trial" with two arms (Figure 1): "Fast-track" (EHPC) versus standard care trial because contamination and poor uptake in the control group are common problems faced by randomized controlled trials of PC services (18). In this wait-list and fast-track design, all patients have the possibility to receive the service after a waiting period (equivalent to waiting time for services provided by a PC unit). There will be a cross-over analysis of the control arm after crossing over to palliative care. This study design has been shown to be feasible by us and large RCTs $(10,19)$.

\section{Outcome measures}

The primary outcome measures of this study include the following:

Health-related quality of life. It will be measured using the McGill Quality of Life (MQOL) Questionnaire (Chinese version). It is specifically designed for PC patients. It is widely accepted and has good construct validity, convergent and divergent validity, test-retest, and interrater reliability in terminally ill patients. Principle analysis has shown that the physical, psychological, existential, and support domains of this scale are all applicable to the Chinese culture (20).

Mood. It will be measured by the Hospital Anxiety and Depression Scale (HADS) (21). The HADS is a selfreporting rating instrument that comprises two parts: the anxiety and depression subscale. The anxiety and depression subscale each comprises seven items that address anxiety and depressive symptoms, respectively. Each item has four graded response options from 0 (absence) to 3 (extreme). The Chinese HADS has well predictive value. It is considered a simple and reliable tool to evaluate psychological illness in patients under palliative care (21).

Caregiver burden. The Chinese version of the Zarit Burden Interview (ZBI) will be used to assess the family caregiver burden (22). The ZBI has 22 items with good internal consistency $(23,24)$.

The secondary outcome measures include the following:

(I) The number of acute admissions.

(II) The number of admissions to the intensive care unit.

(III) Overall survival.

\section{Adverse events}

No major adverse reaction was reported related to this kind of study. The patient and family's emotional aspects will be followed up after the interventions.

The study was conducted in accordance with the Declaration of Helsinki (as revised in 2013). Ethical approval for this study was given by the Institutional Review Board of the University of Hong Kong (No: UW 19-824).

\section{Sample size calculation}

In a one-way ANOVA study, the sample size of 45 patients in each group (i.e., the total sample size of 90 subjects), is required to achieve $80 \%$ power to detect the differences between the groups with a 0.05 significance level, assuming the standard deviation within the group is 25 . This is calculated using PASS 11.0.7. (25). There are currently 100 active patients with MDS and AML at QMH, and 50 new 
patients with MDS and AML are managed in our hospital. Approximately 60 percent of patients fulfill the inclusion criteria. Therefore, at least 50 patients will be recruited in this 24-month study.

\section{Statistical analysis}

Continuous variables are expressed as mean \pm SD unless otherwise specified. Descriptive statistics were used to characterize the socio-demographic and clinical profile of patients. Multiple regressions will be utilized to examine the correlation between different variables and QOL. Wilcoxon signed-rank test and Friedman's analysis of variance (ANOVA) will be used to test for any significant changes in the QOL scores, HADS scores, and ZBI. Baseline differences and final outcomes of the EHPC and convention supportive care groups will be evaluated using the Wilcoxon rank-sum test and Chi-square test. Overall survival survivals will be determined using the Kaplan-Meier method and differences in survival using the log-rank test. Two-tailed $\mathrm{P}$ values $<0.05$ will be regarded as significant. Data will be analyzed with SPSS version 23.0 (Chicago, IL, USA).

\section{Ethics issue}

This trial is carried out in accordance with the Declaration of Helsinki (as revised in 2013) and the International ethical guidelines for biomedical research involving human subjects (26,27). The Hong Kong University/ Hospital Authority Hong Kong West Institutional Review Board (HKU/HA HKW IRB) has approved the study protocol and consent forms. The registration number is UW 19-824. The recruitment started on January 1, 2020, and is expected to be finished on/before July 2022 .

The participants will sign the informed consent form before the trial, and they have the right to withdraw. The committee members will make the final decision on the update the plan of the protocol. We will submit a study completion report upon completion of the clinical study.

\section{Informed consent}

RA would explain to the participants on the study protocol. The assessments and precautions would be briefed to the participants further by the RA. The participants reserve their right to participate/refuse the study. The collected data will not be deleted and will be used for the final analysis if a participant withdraws. A written informed consent from each participant will be obtained prior to the initiation of any study-related treatment. The RA will obtain consent from the participants.

\section{Discussion}

This is the first trial to assess the early integrated palliative care with enhanced psychosocial interventions to both HM patients and their primary family members in Hong Kong. Other research has studied patients with elderly leukaemia/ AML $(28,29)$. Patients with MDS/AML sometimes experience a sudden change of condition or even death (28). An early approach with PC and hematology teams working together to meet the needs of MDS/AML patients might improve their QOL and reduce caregiver burden. The pilot results from this study might lead to major improvements in the care of patients with MDS or AML after disease treatment failure. Significant results from this study may lead to improvements in the care of patients with MDS and $\mathrm{AML}$, and subsequently, result in a reduced requirement for aggressive care.

PC is a multidisciplinary approach that helps to enhance QOL in people when approaching EOL (30). A growing body of literature has identified significant challenges in providing PC in hematological cancer patients (31). Multiple factors (the disease nature, new advances in treatment, the level of care) might complicate the transition to PC. Other barriers for PC integration include limited published research specifically on HM patients' needs, misperceptions about PC as EOL, and lack of a clearly defined model of collaboration $(32,33)$.

The evidence for supporting an effective model of integrated PC service in haematology malignancies is not well known. Our current practice is that the haematologist will be responsible for initiating the topics for referring their patients to PC team while the disease treatment failed. However, it is often too late for the PC referral (3). In this trial, we examine our proposed model and clinical outcomes of an integrated PC approach starting when second line disease treatment failed in a group of MDS/AML patients in RCT. We decided to test this criterion since these are truly refractory patients and, at the same time, able to intercept a wide range of patients and needs. The parent team might sometimes underestimate PC needs and are reluctant to refer patients to a PC service. These problems make this population more homogenous than we could think according to the haematologic diagnosis.

Apart from improving patient's symptoms and mood, 
EHPC might also reduce the caregiver burden of MDS and AML patients in this trial. Previous literature had shown the benefits of PC interventions to assist family caregivers of patients with advanced medical disease in America and other countries (33). And the clinical outcomes of RCTs in cancer (34) and end-stage renal failure (19) also support this kind of PC intervention that could reduce caregiver burden and anxiety. It was also found that the family members were more motivated for the discharge plan and became satisfactory towards the caregiving role (35). Addressing caregivers' concerns and acknowledging the importance of their role is often associated with a positive view of caring (35).

The roles of both PC nurse and social worker in the EHPC program were also important (36-39). The psychosocial needs of the family caregiver are often regarded as not as important as that of the patient. In addition, the caregivers might not be aware of the social support available or a feeling of being ignorant (36). Onsite support to caregivers in our EHPC program could be an effective way to address and solve caregivers' burdens in this regard.

Our trial would have several limitations. First of all, family caregivers in both groups might have variable experiences and perceptions of care due to personal, familial, and economic differences. However, the RCT nature of this study might overcome these differences. Secondly, our study is limited to Hong Kong Chinese MDS/AML patients and hence might not be generalizable to other populations due to social and cultural differences. Last but not least, the study design has incurred substantial costs on manpower, research nurses, and other research-related issues without specific funding support. Our trial will also study reasons for non-adherence, dropouts, and missing data.

\section{Acknowledgments}

We would like to thank all the staff of Haematology Unit in QMH and Palliative Medical Unit in Grantham Hospital for their support to our program.

Funding: None.

\section{Footnote}

Reporting Checklist: The authors have completed the SPIRIT reporting checklist. Available at https://dx.doi. org/10.21037/apm-20-1633

Peer Review File: Available at https://dx.doi.org/10.21037/ apm-20-1633

Conflicts of Interest: All authors have completed the ICMJE uniform disclosure form (available at https://dx.doi. org/10.21037/apm-20-1633). Dr. Kwok Ying Chan serves as an unpaid editorial board member of Annals of Palliative Medicine from Jul 2019 to Jun 2021. The other authors have no conflicts of interest to declare.

Ethical Statement: The authors are accountable for all aspects of the work in ensuring that questions related to the accuracy or integrity of any part of the work are appropriately investigated and resolved. The study was conducted in accordance with the Declaration of Helsinki (as revised in 2013). Ethical approval for this study was issued by the Institutional Review Board of the University of Hong Kong and Hospital Authority Hong Kong West Cluster (HKWC) (No: UW 19-824).

Open Access Statement: This is an Open Access article distributed in accordance with the Creative Commons Attribution-NonCommercial-NoDerivs 4.0 International License (CC BY-NC-ND 4.0), which permits the noncommercial replication and distribution of the article with the strict proviso that no changes or edits are made and the original work is properly cited (including links to both the formal publication through the relevant DOI and the license). See: https://creativecommons.org/licenses/by-nc-nd/4.0/.

\section{References}

1. Thol F, Schlenk RF, Heuser M, et al. How I treat refractory and early relapsed acute myeloid leukemia. Blood 2015;126:319-27.

2. Juliusson G, Antunovic P, Derolf A, et al. Age and acute myeloid leukemia: real world data on decision to treat and outcomes from the Swedish Acute Leukemia Registry. Blood 2009;113:4179-87.

3. Prébet T, Gore SD, Esterni B, et al. Outcome of highrisk myelodysplastic syndrome after azacitidine treatment failure. J Clin Oncol 2011;29:3322-7.

4. Epstein AS, Goldberg GR, Meier DE. Palliative care and hematologic oncology: the promise of collaboration. Blood Rev 2012;26:233-9.

5. Manitta V, Zordan R, Cole-Sinclair M, et al. The symptom burden of patients with hematological malignancy: a crosssectional observational study. J Pain Symptom Manage 2011;42:432-42. 
6. Fadul NA, El Osta B, Dalal S, et al. Comparison of symptom burden among patients referred to palliative care with hematologic malignancies versus those with solid tumors. J Palliat Med 2008;11:422-7.

7. Manitta VJ, Philip JA, Cole-Sinclair MF. Palliative care and the hemato-oncological patient: can we live together? A review of the literature. J Palliat Med 2010;13:1021-5.

8. Ferrell BR, Temel JS, Temin S, et al. Integration of Palliative Care Into Standard Oncology Care: American Society of Clinical Oncology Clinical Practice Guideline Update. J Clin Oncol 2017;35:96-112.

9. Bakitas M, Lyons KD, Hegel MT, et al. Effects of a palliative care intervention on clinical outcomes in patients with advanced cancer: the Project ENABLE II randomized controlled trial. JAMA 2009;302:741-9.

10. Bakitas MA, Tosteson TD, Li Z, et al. Early Versus Delayed Initiation of Concurrent Palliative Oncology Care: Patient Outcomes in the ENABLE III Randomized Controlled Trial. J Clin Oncol 2015;33:1438-45.

11. Dionne-Odom JN, Azuero A, Lyons KD, et al. Benefits of Early Versus Delayed Palliative Care to Informal Family Caregivers of Patients With Advanced Cancer: Outcomes From the ENABLE III Randomized Controlled Trial. J Clin Oncol 2015;33:1446-52.

12. Zimmermann C, Swami N, Krzyzanowska M, et al. Early palliative care for patients with advanced cancer: a clusterrandomised controlled trial. Lancet 2014;383:1721-30.

13. LeBlanc TW, El-Jawahri A. When and why should patients with hematologic malignancies see a palliative care specialist? Hematology Am Soc Hematol Educ Program 2015;2015:471-8.

14. Cheng HW, Li CW, Chan KY, et al. End-of-life characteristics and palliative care provision for elderly patients suffering from acute myeloid leukemia. Support Care Cancer 2015;23:111-6.

15. Roberts C, Torgerson D. Randomisation methods in controlled trials. BMJ 1998;317:1301.

16. Arber DA, Orazi A, Hasserjian R, et al. The 2016 revision to the World Health Organization classification of myeloid neoplasms and acute leukemia. Blood 2016;127:2391-405.

17. Chan KY, Gill H, Chan TSY, et al. Early integrated palliative care for haematology cancer patients-the impact on symptom burden in Hong Kong. Ann Palliat Med 2021;10:6316-24.

18. Higginson IJ, Finlay IG, Goodwin DM, et al. Is there evidence that palliative care teams alter end-of-life experiences of patients and their caregivers? J Pain Symptom Manage 2003;25:150-68.
19. Chan KY, Yip T, Yap DY, et al. Enhanced Psychosocial Support for Caregiver Burden for Patients With Chronic Kidney Failure Choosing Not to Be Treated by Dialysis or Transplantation: A Pilot Randomized Controlled Trial. Am J Kidney Dis 2016;67:585-92.

20. Lo RS, Woo J, Zhoc KC, et al. Cross-cultural validation of the McGill Quality of Life questionnaire in Hong Kong Chinese. Palliat Med 2001;15:387-97.

21. Leung CM, Wing YK, Kwong PK, et al. Validation of the Chinese-Cantonese version of the hospital anxiety and depression scale and comparison with the Hamilton Rating Scale of Depression. Acta Psychiatr Scand 1999;100:456-61.

22. Zarit SH, Reever KE, Bach-Peterson J. Relatives of the impaired elderly: correlates of feelings of burden. Gerontologist 1980;20:649-55.

23. Arai Y, Kudo K, Hosokawa T, et al. Reliability and validity of the Japanese version of the Zarit Caregiver Burden interview. Psychiatry Clin Neurosci 1997;51:281-7.

24. Chan TSF, Lam LCW, Chiu HFK. Validation of the Chinese version of the Zarit Burden Interview. Hong Kong Journal of Psychiatry 2005;15:9-14.

25. Kimmel PL, Emont SL, Newmann JM, et al. ESRD patient quality of life: symptoms, spiritual beliefs, psychosocial factors, and ethnicity. Am J Kidney Dis 2003;42:713-21.

26. Issue Information-Declaration of Helsinki. J Bone Miner Res 2018;33:BM i-BM ii.

27. Mathur R, Swaminathan S. National ethical guidelines for biomedical \& health research involving human participants, 2017: A commentary. Indian J Med Res 2018;148:279-83.

28. Barbaret C, Berthiller J, Schott Pethelaz AM, et al. Research protocol on early palliative care in patients with acute leukaemia after one relapse. BMJ Support Palliat Care 2017;7:480-4.

29. Button E, Chan RJ, Chambers S, et al. A systematic review of prognostic factors at the end of life for people with a hematological malignancy. BMC Cancer 2017;17:213.

30. Callaway MV, Connor SR, Foley KM. World Health Organization Public Health Model: A Roadmap for Palliative Care Development. J Pain Symptom Manage 2018;55:S6-S13.

31. LeBlanc TW, Roeland EJ, El-Jawahri A. Early Palliative Care for Patients with Hematologic Malignancies: Is It Really so Difficult to Achieve? Curr Hematol Malig Rep 2017;12:300-8.

32. Auret K, Bulsara C, Joske D. Australasian haematologist referral patterns to palliative care: lack of consensus on 
when and why. Intern Med J 2003;33:566-71.

33. Cheng HWB, Lam KO. Supportive and palliative care in hemato-oncology: how best to achieve seamless integration and subspecialty development? Ann Hematol 2021;100:601-6.

34. McMillan SC, Small BJ, Weitzner M, et al. Impact of coping skills intervention with family caregivers of hospice patients with cancer: a randomized clinical trial. Cancer 2006;106:214-22.

35. Monin JK, Schulz R. Interpersonal effects of suffering in older adult caregiving relationships. Psychol Aging 2009;24:681-95.

36. Schulz R, Hebert RS, Dew MA, et al. Patient suffering

Cite this article as: Chan KY, Gill H, Li CW, Chan TSY, Au HY, Wong CY, Tsang KW, Lo RSK, Cheng BHW. Impact of enhanced haematology palliative care services in patients with myelodysplastic syndrome and acute myeloid leukaemia: study protocol for a randomized controlled trial. Ann Palliat Med 2021;10(9):10013-10021. doi: 10.21037/apm-201633 and caregiver compassion: new opportunities for research, practice, and policy. Gerontologist 2007;47:4-13.

37. Chan KY, Yap DYH, Yip T, et al. Palliative Care Consultation in Advanced Chronic Kidney Disease with Pain. J Palliat Med 2018;21:809-14.

38. Chan KY, Yip T, Yap DYH, et al. A Pilot Comprehensive Psychoeducation Program for Fluid Management in Renal Palliative Care Patients: Impact on Health Care Utilization. J Palliat Med 2020;23:1518-24.

39. Chan KY, Chiu HY, Yap DYH, et al. Impact of structured advance care planning program on patients' wish items and healthcare utilization. Ann Palliat Med 2021;10:1421-30. 